\title{
Survival Status and Its Associated Factors among Under- Five Children Admitted with Complicated Severe Acute Malnutrition in Hospitals of Wolaita Zone, South Ethiopia: Retrospective Cohort Study
}

\author{
Amare Admasu ${ }^{1 *}$, Elazar Tadesse ${ }^{2}$, Tezera Moshago ${ }^{3}$ and Niguse Mekonnen ${ }^{4}$ \\ ${ }^{1}$ Clinician at Wolaita Sodo University Teaching Referal Hospital, Woliata Sodo Zone, Ethiopia \\ ${ }^{2}$ Metropolitian University, Addis Ababa, Ethiopia \\ ${ }^{3,4}$ Wolaita Sodo University, School of Public Health, College of Health Sciences and medicine, Wolaita Sodo, Ethiopia
}

Received: 12 June, 2017; Accepted: 30 June, 2017; Published: 06 September, 2017

*Corresponding author: Amare Admasu, Clinician at Wolaita Sodo University Teaching Referal Hospital, Woliata Sodo Zone, Ethiopia, Tel:+251910178276 or 0916746544; E-mail: amareadmasu2@gmail.com

\begin{abstract}
Background: Complicated severe acute malnutrition is the common reason for pediatric hospital admission in many poor countries, which pauses additional burden on limited resources. In hospitals, it remains poorly managed which led to mortality rate of under-five children became higher than the acceptable level as different studies revealed. However, survival status and its associated factors for complicated severe acute malnutrition were yet not get attention to halt its sequels.

Objective: This study aims at assessing survival status and its associated factors among under-five children admitted with complicated severe acute malnutrition to stabilization centers of Hospitals in Wolaita Zone.

Method: A retrospective cohort study comprised of 340 under-five children admitted for treatment of complicated severe acute malnutrition in two hospitals of Wolaita Zone in past 39 months. From the study period, the data were collected using the structured checklist, then entered and cleaned by Epi info version 3. 5. 4 and analyzed by SPSS version 20. Descriptive statistics was used to summarize child characteristics and treatment outcomes; cure rate, death rate, defaulters, and non-responders. Variables that were having P-value $<0.25$ in bivariate analysis were entered into a multivariable Cox- proportional regression model to identify the predictors of mortality. Level of statistical significance was declared at p-value $<$ 0.05 .

Results: From a total of 30 deaths occurred at SC 13, 8, and 6 deaths occur within 72 hours, 4-6 days and 7-9 days after admission respectively. The majority of death $63.3 \%$ occurred in children age $<12$ months and the same magnitude $10 \%$ for 13-24 and 23-36 months of age groups. Being septic at admission with their respective $95 \%$ confidence interval were $2.9(1.03,8.40)$, being hypothermic $11.8(3.77,37.02)$ and not giving antibiotics at admission $3.7(1.55,8.64)$ were an independent predictors of death.

Conclusion: Preventing hypothermia, treating sepsis and providing antibiotics at admission has a major effect in saving the life of children with complicated severe acute malnutrition in the stabilization centers. Thus, special attention should be gven for children with hypothermia, sepsis and provision of antibiotics for further reduction of death within the first few days of admission is faramaunt important.
\end{abstract}

Keywords: Complicated Severe Acute Malnutrition; Mortality Rate;

\section{Introduction}

At the hospital level, training of health care workers in World Health Organization (WHO) treatment guidelines are part of the policy to manage complicated SAM in the inpatient setting [1]. Global trends show that malnutrition contributes to over half of all under-five deaths [2]. In Bangladesh study conducted exhibited $50 \%$ of deaths were within forty-eight hours of admission. Having malaria, severe anemia, and Tuberculosis (TB), as co-infection with severe acute malnutrition were found to be an independent predictor of mortality. Similarly; hypothermia, septicemia, and pneumonia were realized as risk factors for death [3]. The study conducted in South Africa showed that Case-fatality rate for SAM cases is remaining high $40.1 \%$ [4]. Likewise, another finding from Zambia revealed mortality rate $51 \%$ Vs 35\% from 20092013 [5]. Thus, there needs exist for improved adherence to the WHO guidelines and periodic clinical audits to reduce death from childhood malnutrition [6].

Ethiopia is one of the countries with highest under-five child mortality rate, with malnutrition underlying to $57 \%$ of all children's deaths [7]. The urban-rural difference in mortality 
rates is more pronounced in the case of under-five year aged children. Under-five mortality rates range from a low of 53 per 1,000 live births in Addis Ababa to a high of 169 per 1,000 live births in Benishangul-Gumuz. Under-five mortality is also relatively high in Afar, Gambella and Somalia [8]. Likewise, in southwest and south Ethiopia, studies revealed that the recovery rate and defaulter rate were remote from the international acceptable standard ranges [9]. Despite the existence of inpatient and other nutrition programs in every corner of Ethiopia, the national survey and different studies, have shown that deaths due to severe acute malnutrition are indicated to be still high $[8,10]$.

Recently, research conducted in Jimma and Eastern Ethiopia has shown that the death rate was $9.3 \%(27.3 \%$ in the first 48 hours and $60.2 \%$ by the end of the first week) and $13 \%$ respectively $[11,12]$. The first week of inpatient stay was the most critical to the survival of children; most deaths occurred during this period $[11,13]$. Having co-morbidities prior to the admission, development of cross infection during the stay, being critical at arrival, lack of regular and supportive supervision, lack of training for TFU staffs regarding the guideline for the management of SAM and high staff workload during the night affects the treatment outcome. These are the potential contributors that lead to inappropriate management and increased death rate [14]. The dissimilarities of mortality and survival status in different study settings may be related to variation in quality of service delivery.

Even though enormous problems determine survival status, limited studies were done in the country and no evidence found mainly in the study settings. Therefore, the study intended to assess the survival status and it associated factors among underfive children admitted with complicated SAM to SC is public health important problem in Ethiopia especially in the study settings with limited resources; the burden is more than anticipated.

\section{Methodology}

\section{Study Setting}

The study was conducted in two Hospitals of Wolaita zone, Southern Ethiopia. Wolaita Zone situated at $165 \mathrm{Km}$ from Regional Capital City, Hawassa, at $6^{\circ} 49^{\prime} \mathrm{N}$ latitude and $39^{\circ} 47^{\prime} \mathrm{E}$ longitude, at an altitude of about $1900 \mathrm{~m}$ and $387 \mathrm{Km}$ from Addis Ababa. This Zone is one of the most densely populated areas in the country with a density of 290 people per square kilometer. According to the 2007 census, According to the Zone had an estimated population of 1,882,833 from this about 922,588 are males in 2015 [15]. There are an estimated 293,910 underfive children. Concerning the study area location Wolaita Sodo University Teaching Referral Hospital (WSUTRH) which is one and the only governmental referral hospital in Wolaita zone providing SC service and is located in the Sodo town, whereas Dubbo St. Marry Primary Hospital, which is a private organization located at Boloso Sore Woreda, Areka town administration where there is a functional SC center.

Regarding study settings WSUTRH from a total of 255 functional beds, of them 70 beds are reserved for pediatric age groups. Total inpatient admission in the last year is 8,762 and the bed occupancy rate was $74.5 \%$. Meanwhile, the disease burden SAM is the 8th top cause of under-five year admission [16]. Similarly, in Dubbo St. Marry Primary Hospital had 103 functional beds of them 33 were reserved for pediatric age groups [17].

\section{Study Design and Period}

A retrospective cohort study design was used from April 1st, 2016 to April 30th, 2016.

\section{Source Population}

Records of all children admitted to pediatric wards with severe acute malnutrition during the study period.

\section{Study Population}

All under-five children admitted with complicated SAM to stabilization centers of both hospitals.

\section{Inclusion and Exclusion Criteria}

Inclusion criteria: Children fulfill criteria to diagnose complicated SAM and admitted. Children who admitted more than once were assessed only for the last admission only.

Exclusion criteria: Incomplete records of c children's chart with incomplete data of age and/or sex. Children whose treatment outcome not recorded and children whose admission date and discharge date not recorded.

\section{Sample Size and Sampling Procedures}

All eligible children admitted to the SC from January $1^{\text {st }}, 2013$ to March $30^{\text {th }}, 2016$ based on inclusion and exclusion criteria were studied.

\section{Variables}

Dependent variable: Death

Event: death; Times to the event: time to death from admission till death happen.

\section{Independent variables}

Child characteristics: age, sex

Residence: Urban and Rural

Clinical conditions: vomiting, dehydration, edema

Co-morbidities: the presence of one or more additional disease or co-occurrence with primary disease.

Treatment Outcome: weight gain, defaulter, non-responded, absconded, death, cure

\section{Types of severe}

Acute malnutrition: marasmus, kwashiorkor, marasmus-kwash

Medication: routine antibiotic (amoxicillin, Vit. A, folic acid, deworming, anti-malaria, ReSoMal) and special medication (IV fluid, IV antibiotic, blood transfusion) 


\section{Data Collection Instruments}

A checklist was developed from the standard treatment protocol for the management of severe acute malnutrition, SAM registration log book, SAM monitoring multi-chart and reviewing related literatures to collect the required individual information from the relevant documents (Figure 1).

The checklist consists of the following information: Patient related data (age, sex, and residence), Co-morbidities/infection, types of severe acute malnutrition, feeding phase and types of feeding, frequency of feed and amount per feed, medication given, and health information on different topics, Condition at discharge and date and time of death.

\section{Data Collection and Quality Assurance Procedures}

Primarily pre-structured checklist was developed by adapting variables from different relevant literatures and SAM management guidelines. Then card numbers were collected from pediatric ward log books. Next, the card numbers were crosschecked to avoid repetition and sent to the card room access cards. Prior to data collection two day intensive training was given to 4 professional (Bsc holder) data collectors and 1 review professional supervisors who have previous experience by data reviewing and supervision. All data collectors were from other facilities to control bias. Prior to actual data collection, pre-test was done by $5 \%$ of collected cards for the actual survey in months not included in the current study and then necessary correction was taken accordingly.

To avoid repetition of reviewing a single card attached with its checklist until the principal investigator verify the completeness and put a unique mark to prevent recurring review and kept it in separately until the compilation of the data collection period. Charts with more than one round admission were reviewed only for latest readmission and for it only because one chart reviewed only once. Data was extracted first from children's registers and then from records (card and multi-chart). Collected data were sorted and checked for errors and completeness on site daily by supervisors. Finally, data from two sources was linked by patient's card number.

\section{Operational definitions}

Censoring: Those cases as defaulters, recovered or none recovered.

Complete record: if age in months, sex of the child, admission date and time, type major complications, discharged date and treatment outcome recorded.

Complicated SAM: Children who are acutely malnourished with associated medical complications and/or poor appetite; and infants less than 6 months with SAM

Co-morbidities: In children with severe acute malnutrition, having TB, and/or HIV and/or malaria and/or pneumonia, and/ or diarrhea, and/or severe anemia co-infection on admission to stabilization center.

Cured: Patient that has reached the discharge criteria with improvement

Dead: Patient that has died whiles he/ she was in the programme stabilization center.

Defaulter: Patient that is absent for 2 consecutive weighing (2 days in inpatient).

Hyperthermia: Defined when the body axillary temperature is $\geq 38.5^{\circ} \mathrm{C}[18]$.

Hypothermia: Defined when the body axillary temperature is below $35^{\circ} \mathrm{C}[18]$.

Inpatient Treatment/ care: Children who are acutely malnourished with associated medical complications and/ or poor appetite; and infants less than 6 months with the SAM need to be treated in an inpatient care facility until they are well enough to continue nutritional rehabilitation in OTP.

Non-responder: Patient that has not reached the discharge criteria after 40 days in the inpatient program $[18,19]$.

Phase 1 (Stabilization phase): children with complicated SAM are initially admitted to an inpatient facility for stabilization. In this phase: Life-threatening medical complications are treated, Routine drugs are given to correct specific deficiencies, Feeding with F-75 milk (low caloric and sodium) is begun [18]

Sepsis: The presence of bacteria (bacteremia), other infectious organisms, or toxins created by infectious organisms in the bloodstream to spread throughout the body [20].

Severe Anemia: If the hemoglobin concentration is less than $40 \mathrm{~g} / \mathrm{l}$ or the packed-cell volume is less than $12 \%$ the child has very severe anemia.

Phase 2 (Rehabilitation Phase): Children that progress through phase 1 and transition phase enter phase 2 (rehabilitation phase) when they have a good appetite and no major medical complication. In this phase: Routine drugs are continued, Feeding with RUTF or F100 is started [18].

Survival: those who are alive at discharge from stabilization centers.

Time to event: Death is the event of interest. Time to event was calculated in days by subtracting the date of admission from the date of event occurred in under-five children admitted with severe acute malnutrition to stabilization centers of study settings during the study period.

Transition phase: Once the child appetite recovers and the main medical complications are under control and edema start to reduce, a transition phase is started where F-100 or RUTF (Ready-to-Use Therapeutic Food) is introduced [18].

Under-five children: age of 0-59 months

\section{Data Management and Analysis Procedures}

Data was entered into Epi info version 3. 5. 4 exported to SPSS version 20 for Windows and cleaned to check for completeness, extreme and missing values. All statistical analyses were done using SPSS version 20 for windows. Univariate (descriptive) 


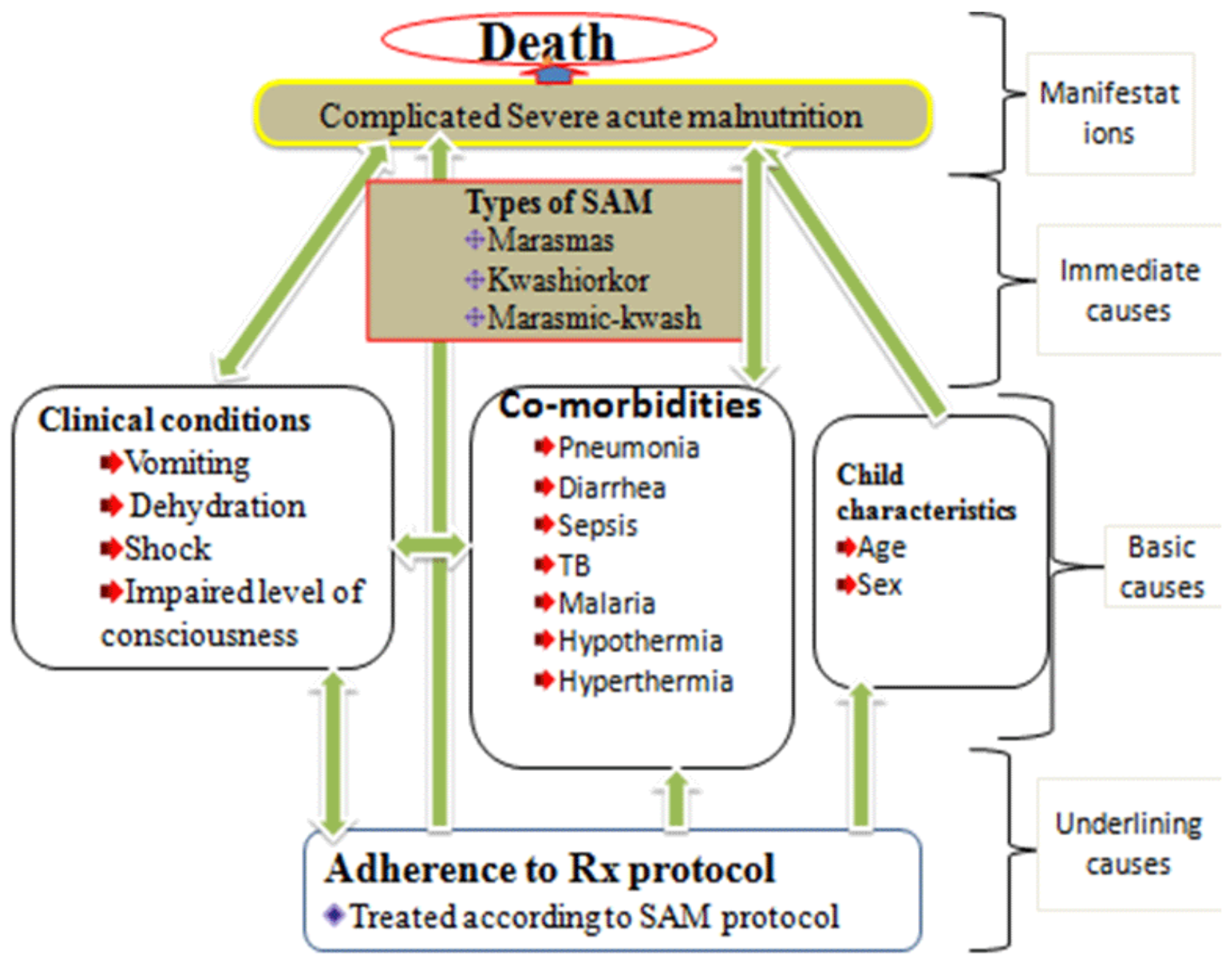

Figure 1: Schematic presentation of mortality predictors from reviewing related literatures

analyses were performed and presented by tables and graphs. Chisquare test was conducted to determine if there are adequate cell counts for each categorical variable. Kaplan-Meier technique was used to compare survival experience by different category. Cox proportional hazards regression model was used to determine predictors of mortality. Before modeling, Cox regression model assumption of proportional hazards was checked by KaplanMeier hazard plots and testing an interaction of covariate with time. Multicollinearity among independent variables was checked for variance inflation factor and it was less than 10 .

Multivariable Cox regression was preceded by bivariate Cox regression during modeling. Variables with $\mathrm{p}<0.25$ by bivariable analysis and missing information either (present/ absent) of specific variables not more than $5 \%$ were considered as candidate for multivariate cox-regression. Multivariable Cox regression was run using the Forward Wald method to identify best independent predictors of death. The possibilities of interactions (effect measure modification) among independent variables were explored by including interaction terms in the multivariable Cox regression. P-value of less than 0.05 at $95 \%$ confidence interval was considered as a statistical significance to identify independent predictors of earlier death in multivariable analysis. Hazard Ratio (HR) was used as a measure of association.

\section{Ethical Consideration}

The ethical clearance was obtained from the Ethical Review Committee of Wolaita Sodo University college of Medicine and Health Science then agreement to use the data was obtained from study settings. Confidentiality was assured of collecting data namelessly using just the card number of each record. Since the study was conducted through review of medical records, there is no potential harm to individual patients. 


\section{Result}

A total of 483 children's were admitted to stabilization centers of WSUTRH and Dubbo St. Marry Primary Hospitals during the follow-up period. Of these, 238 were at WSUTR where 12 charts with uncomplicated SAM diagnosis, 16 charts of age ineligibility, 10 charts of the unknown outcome at discharge and 34 unavailable charts were excluded. From Dubbo hospital, a total of 245 SAM cases, 4 charts of uncomplicated SAM, 29 charts of age ineligibility, 5 charts of the unknown outcome at discharge and 33 unavailable charts were excluded. Therefore, from the total 483 children charts reviewed, 143 were excluded. A total of 340 charts fulfilled inclusion criteria; 166 (48.8\%) charts from WSUTRH and 174(51.2\%) from Dubbo (Figure 2).

The majority 320 (94.1\%) of children were newly admitted, $16(4.7 \%)$ readmission and $4(1.2 \%)$ were others. Regarding admission criteria $71(20.9 \%)$ were $\mathrm{WFH}<70 \%, 106(31.2 \%)$ edematus, $86(25.3 \%)$ by MUAC and severe wasting were $58(17.1 \%)$. More than half of children's under the cohort had no recorded information on breast fed $(52.9 \%)$ and complementary feeding (57.1\%). About three folds of children 252(74.1\%) had no record of the amount per feed on the therapeutic milk. In the majority of charts reviewed; mothers or caregivers 277 (66.8\%) have no recorded information, whether they had received a health nutrition education at discharge or not and it was given only for $88(25.9 \%)$. Thus, these variables were excluded from final analysis.

\section{Socio-Demographic Characteristics of the Cohort}

Of the 340 children included in the study, 179(52.6\%) were male and $234(68.8 \%)$ were from a rural area. From a total of 30 deaths in the SC, 23 children died were from a rural area They are three and more times higher than urban. Regarding age distribution, $79(23.2 \%)$ were less than 6 months and 140 $(41.2 \%) \geq 24$ months of age. The median age of the children at admission was 12 months with IQR (interquartile range) of 7 to 36 months (Table 1).

\section{Mortality among Complicated SAM}

From a total of 340 study subjects 30 children were dying in the SC. Of them 15/166 (9.04\%) were from WSUTRH and the rest $15 / 174(8.62 \%)$ were from DSMPH. There was no difference in the incidence of deaths between two sexes. The majority of death $63.3 \%$ occurred in children age less than 12 months and the same magnitude $10 \%$ for $13-24$ and $23-36$ months of age groups. The remaining $6.7 \%$ and $10 \%$ of death occurred among 37-48 and above 49 months of age respectively. In addition, more than 3/4th of death occurred in children who come from rural area. This may be children from rural area were either more critical or women who care for them were less educated to follow instructions from medical care providers compared with urban. The incidence of death was 7.2 per 1000-person day throughout the study period. The greatest portion of death occurs within 3 days of admission $13 / 30(43.3 \%)$ and only $3(10.0 \%)$ died after 10 days (Figure 4$)$.

\section{Co-Morbidities at Admission}

The greater part of $158(46.5 \%)$ children admitted to SC had diarrhea at admission, followed by pneumonia 107(31.5\%), malaria $65(19.1 \%)$, tuberculosis $44(12.9 \%)$ and $7(2.1 \%)$ hypothermia (Table 2). However, none of them had statistically significant association with early death except sepsis and hypothermia.

\section{Feeding and Routine Medications}

In Phase I F-75 given in 281(82.6\%), diluted F-100 for 52 $(15.3 \%)$ and $\mathrm{F}-100$ for $2(0.6 \%)$. In a transition phase $\mathrm{F}_{-} 75$ for $7(32 \%)$, diluted F-100 for 28(8.2\%), F_100 194(57.1\%), Phase II F_75 2(0.6\%), diluted F-100 20(5.9\%), F_100 193(56.8\%). This shows that the despite the instructions of complicated SAM management guideline of Ethiopia $0.6 \%$ of children in phase I and II and 32\% at transition phase were treated against the coaching of SAM protocol. Even though, assessing missed management in children with complicated SAM was it is out of the scope of the study, this may be due to lack of regular training for TFU staffs, regular monitoring and evaluation system and irregularities in therapeutic milk supply [21].

Regarding routine medications; more than half of children $175(51.5 \%)$ were given antibiotics; $166(48.8 \%)$ Vitamin A and folic acid $5 \mathrm{mg}$ in $161(47.4 \%)$. Ninety-seven $(28.5 \%)$ were Iv-infused (resuscitated with IV fluid), RESOMAL was given in 108(31.8\%), NG-tube for feeding inserted for 154(45.3\%), and $18(5.3 \%)$ were transfused with whole blood, while, deworming at Phase II was not given in 225(66.2\%). Similarly, some medications that national SAM management guideline recommends to supply or treat complicated SAM were not implemented for some of the cases. Thus, this may also be due to irregular of supply or adherence to treat SAM may not supervised by external bodies.

\section{Types of Complicated Severe Acute Malnutrition}

Of 340 children's records reviewed; more than half $209(61.5 \%)$ had marasmus, $112(32.9 \%)$ had kwashiorkor, and $19(15.6 \%)$ had marasmic-kuwash. Regarding mortality,

\begin{tabular}{|c|c|c|c|}
\hline Variables & $\begin{array}{c}\text { Categories of } \\
\text { variables }\end{array}$ & Number & (\%) \\
\hline \multirow{3}{*}{ Age } & $<6$ months & 79 & 23.2 \\
\hline & 6-23 months & 121 & 35.6 \\
\hline & $\geq 24$ months & 140 & 41.2 \\
\hline \multirow[b]{2}{*}{ Sex } & Male & 179 & 52.6 \\
\hline & Female & 161 & 47.4 \\
\hline \multirow[b]{2}{*}{ Residence } & Rural & 234 & 68.8 \\
\hline & Urban & 106 & 31.2 \\
\hline \multirow{2}{*}{$\begin{array}{c}\text { Referrals } \\
\text { from }\end{array}$} & $\begin{array}{c}\text { Health center/ } \\
\text { Hospital }\end{array}$ & 77 & 22.6 \\
\hline & $\mathrm{HP} /$ community & 263 & 77.4 \\
\hline
\end{tabular}




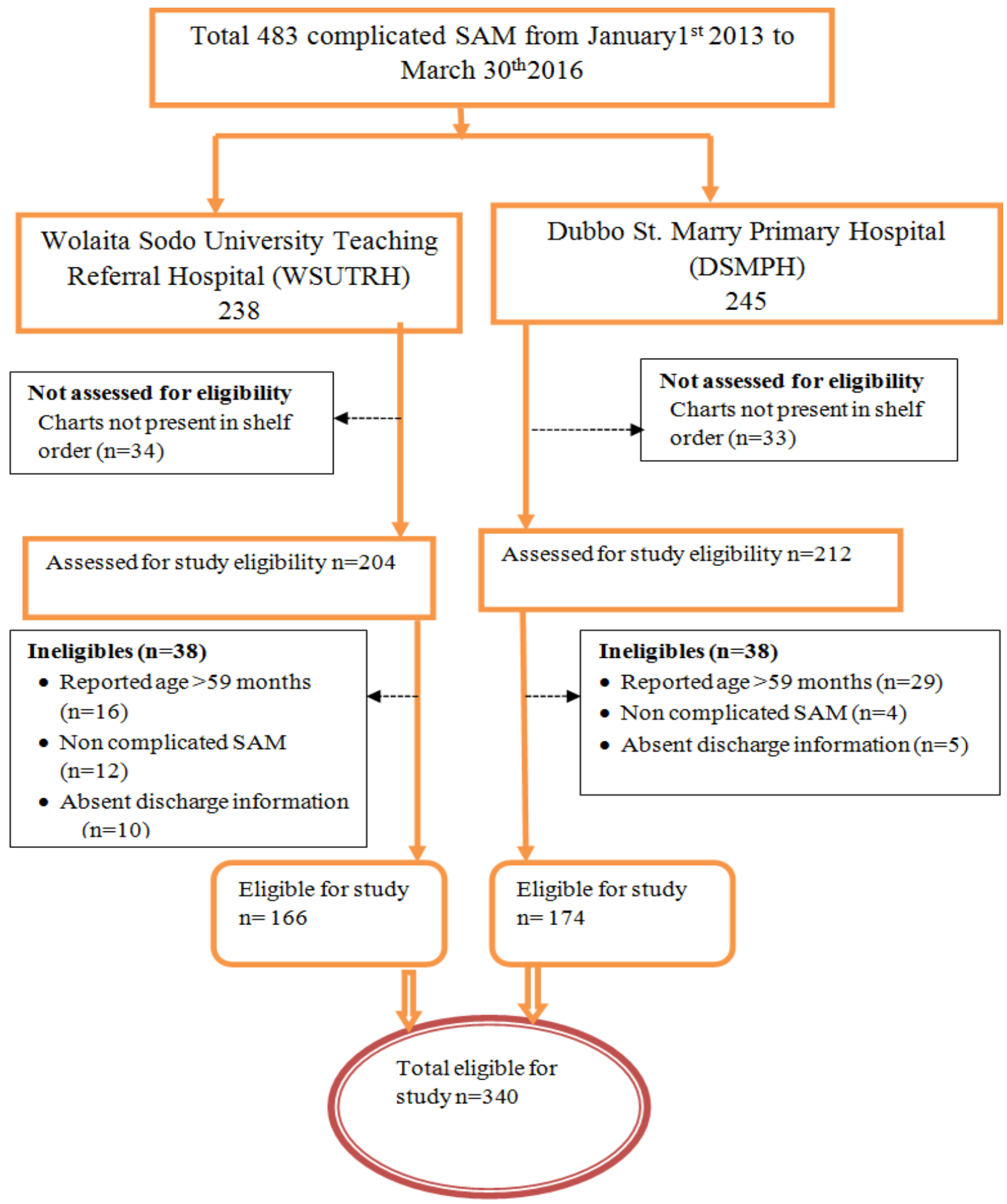

Figure 2: Participants flow of complicated SAM cases admitted to stabilization centers in WSUTRH and DSMPH. 
greatest proportion $21(70 \%)$ of death occurred in children with marasmus whilst kwashiorkor and marasmic-kuwash 7(23.3\%) and $2(6.7 \%)$ respectively.

\section{Treatment Outcomes}

Among 340 children whose records were reviewed, 257(75.6\%) were cured, 30(8.8\%) died, 34(10.0\%) absconded (self-discharged) and 6(1.8\%) were non-responders (Figure 4).

The result of treatment outcome showed that cure rate and mortality rate were around the lowest and highest margin of acceptable level of the national SAM management guideline for inpatient care respectively. Both of these conditions need more attention to reverse; thus, this finding is alarming result to work hard and monitor performance [18] (Table 3).

\section{Clinical Conditions}

The most prevalent clinical condition in children included in our study was dehydration 215(63.4\%) followed by vomiting 159 (46.8\%) and edema 131(38.5\%). Despite the fact that, children having these conditions were not small figures in our study even though none of them were statistically associated with early death.

The largest proportion (29.4\%) of discharge was occurred in the first and (43.8\%) second weeks of admission; with an average

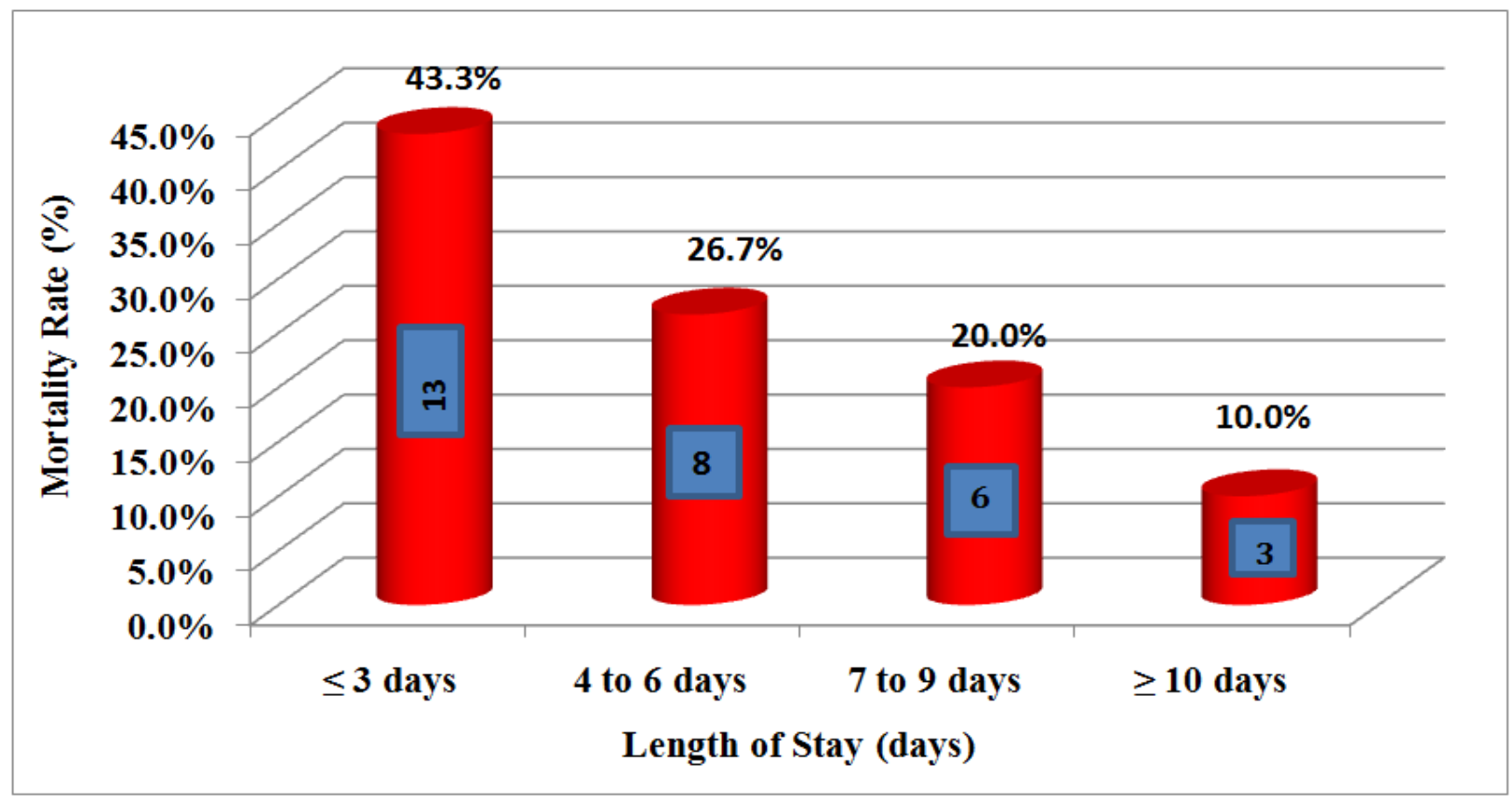

Figure 3: Time to death of children admitted to SC in Wolaita zone, from January 1, 2013 to March 30, 2016(n=30).

Table 2: Co-morbidities in children admitted to stabilization centers of Wolaita zone, from January 1, 2013 to March 30, 2016(n=340).

\begin{tabular}{|c|c|c|}
\hline Variables & Number & (\%) \\
\hline Malaria at admission & 65 & 31.5 \\
\hline Pneumonia at admission & 107 & 46.5 \\
\hline Diarrhea at admission & 158 & 7.6 \\
\hline Sepsis at admission & 26 & 0 \\
\hline Hypothermia & 7 & 0 \\
\hline Hyperthermia & 17 & 12.9 \\
\hline Tuberculosis & 44 & 8.5 \\
\hline Anemia & 29 & 5.3 \\
\hline Impaired level of consciousness & 18 & \\
\hline Skin lesion & 27 & 7.9 \\
\hline
\end{tabular}

Citation: Admasu A, Tadesse E, Moshago T, Mekonnen N. (2017) Survival Status and Its Associated Factors among Under-Five Children Admitted with Complicated Severe Acute Malnutrition in Hospitals of Wolaita Zone, South Ethiopia: Retrospective Cohort Study. 
weight gain of $15.3 \mathrm{~g} / \mathrm{kg} /$ day. The median (IQR) duration from admission to discharge with improvement was 11 days.

\section{Predictors of Mortality in Children Admitted to Stabilization Center}

The following variables were selected as candidates for milt-variable cox-regression during bivariate analysis. Of comorbidities (hypothermia, hyperthermia, and sepsis), medications (antibiotics) and from clinical conditions (shock, Childs' impaired level of consciousness, vomiting, and dehydration) were incorporated in the multivariate cox- regression. In multivariable cox-regression analysis with the significance level at 95\% CI and p-value $<0.05$ were considered as an independent predictor of mortality in children aged 0-59 months with complicated SAM admitted to stabilization centers.

Children with hypothermia, had 11.8 times increased risk of dying when compared to children with complicated SAM with normal temperature (AHR=11.8, 95\% CI [3.77-37.02], $P<0.0001$ ). Similarly, children having sepsis at admission had 2.9 times more at risk of death when compared to those with no sepsis (AHR=2.9, 95\% CI [1.03-8.40], $\mathrm{P}=0.045$,) (Table 4). In addition, regarding routine medications; children who did not have antibiotics at admission 3.7 times more at risk of death (AHR=3.7, 95\% CI [1.55-8.64], $\mathrm{P}=0.003$ ) compared to those had had it (Table 4). The cumulative probability of survival by the end of the $3^{\text {rd }}, 6^{\text {th }}$ and the $9^{\text {th }}$-day was $94 \%, 92 \%$, and $91 \%$ respectively. The probability of survival decline by $2 \%$ for the second three days, then by $1 \%$ for next three days. Thus, the risk of death was very high within the first few days of hospital stay(Figure 5).

The probability of survival by the end of 3rd, 6 th, and the 9th-day for age groups less than 6 months was $95 \%, 88 \%$, and $88 \%$; for 6 to 23 months $94 \%, 92 \%$ and $89 \%$; and greater than 24 months was $93 \%$ for three respective days. These realize that the probability of survival was very low among young age groups than older ones.

The probability of survival by the end of the 3nd , 6th and 9th day children from rural was $93 \%, 90 \%$, and $90 \%$, whereas, $96 \%, 96 \%$, and $93 \%$ for urban respectively (Figure 6). Thus, even though it has no significant association with mortality, especial attention has to be given for younger children and for rural residents who admitted to hospitals with complicated SAM.

\section{Discussion}

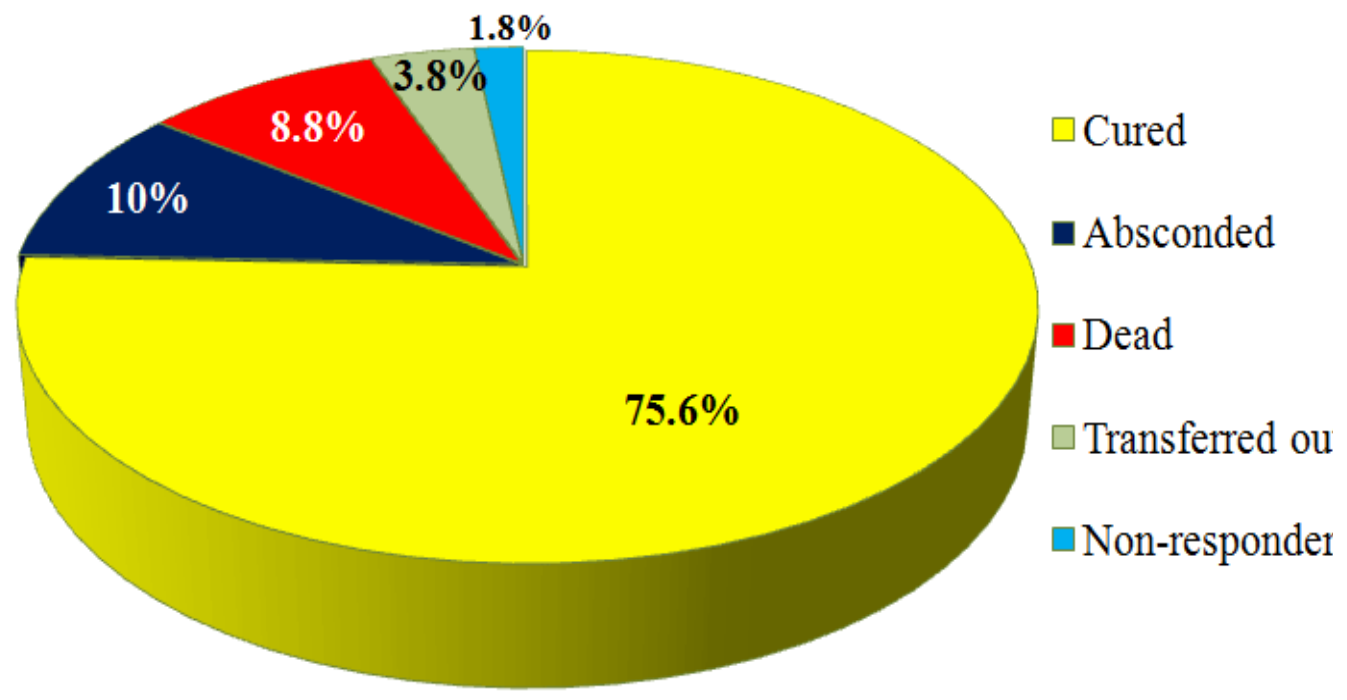

Figure 4: Treatment outcome of children admitted to SC in Wolaita zone, from January $1^{\text {st }}, 2013$ to $M a r c h ~ 30^{\text {th }}, 2016(n=340)$.

From the total of 340 children having complicated SAM included in the study from January $1^{\text {st }}, 2013$ to March $30^{\text {th }}, 2016$; $257(75.6 \%)$ recovery, $30(8.8 \%)$ death, $34(10 \%)$ self-discharge/ defaulters, $13(3.8 \%)$ transfer out and $6(1.8 \%)$ non-responders. In addition, factors identified as having a statistically significant association with the risk of mortality in the study were sepsis, hypothermia, and antibiotics both by bivariate and multivariable cox-regression.

Regarding the type of SAM, marasmus was the most prevalent $209(61.5 \%)$ one type of SAM. This finding is in line with the study done in Dhaka city of Bangladesh (61\%) [22]. But the result was lower than a study conducted in southern Ethiopia were (47\%) of the cases were marasmic [23]. Edematous type of SAM was $131(38.5 \%)$ in the study and it was higher than the study done in Niger $(15 \%)$ but by far lower than the study done in Jimma $(57.2 \%)$ and Southern Ethiopia (53\%) of the participants were having nutritional edema $[2,11,24]$. This may be due to the difference in socio-economic and cultural practices in different parts of the study areas. Diarrhea was the most prevalent 162 (47.6\%) co-morbidity in SAM cases under the study followed by pneumonia, dehydration, malaria, clinical form of TB, severe anemia and hyperthermia with their respective prevalence being (31.5\%), (21.2\%), (19.1\%), (12.9\%), (8.5\%) and (5.0\%). But none of these variables were statistically significant predictors of 
Table 3: Comparison of the treatment outcomes of complicated severe acute malnutrition in children at study settings with Inpatient Care (IPC) acceptable performance range of Ethiopian SAM management guideline

\begin{tabular}{|c|c|c|c|}
\hline \multirow{2}{*}{ Outcome indicator } & Current Outcome & \multicolumn{2}{|c|}{ SPHERE standards } \\
\cline { 2 - 3 } & (\%) & $>75 \%$ & $<$ Alarming \\
\hline Recovery rate & 75.6 & $<15 \%$ & $>25 \%$ \\
\hline Defaulter rate & 10 & $<10 \%$ & $>15 \%$ \\
\hline Case Fatality Rate (CFR) & 8.8 & $\geq 8$ & $<8$ \\
\hline Average weight gain (g/kg/day) & 15.3 & $<4$ weeks & $>6$ weeks \\
\hline Average length of stay (weeks) & 1.8 & & \\
\hline
\end{tabular}

mortality.

The study result revealed that $257(75.6 \%)$ children admitted with complicated SAM to the SC were cured. The percentage of recovered children were higher when compared to the percentage in a prospective cohort conducted in Zambia (53.7\%), Gonder University hospital 68.5\%, North Ethiopia (46.5\%), North West Ethiopia (68.5\%) and lower than the result of the study done in Jimma University Specialized Hospital (77.8 \%)[11,25,26,27] These dissimilarities may be due to variation in adherence and utilization of complicated SAM guideline, differences in SC setups and distance from health facility [27].

The overall mortality rate of the cohort is $8.8 \%$. Many studies conducted in different countries; in Zambia (40.1\%), Tanzania
(13.7\%), North Ethiopia (28.7\%), and North West Ethiopia $(11.7 \%)$ were shown higher incidence of mortality [22, 24, 26, 27]. The study result is comparable with the study reports of death at Sc from Southwestern Ethiopia (9.3\%) [11]. this may be due to variation in the study settings, socioeconomic, maternal or caregivers commitment to take care accordingly and or literacy level [28].

However, the cure rate and mortality rate were in the margin of minimum acceptable of SAM guideline; the percentage of selfdischarge, average weight gain and length of hospital stay of children in the SC of the study were also efficiently harmonizing with national SAM management guideline. The percentage of children who died (8.8\%) was also in line with the minimum

Table 4: Bivariate and multivariate cox-regression analysis for predictors of mortality from complicated SAM in under-five children at SC on admission, Wolaita zone, from January $1^{\text {st }}, 2013$ to March $30^{\text {th }}, 2016(n=340)$

\begin{tabular}{|c|c|c|c|c|c|}
\hline \multirow{2}{*}{ Variables } & \multicolumn{2}{|c|}{ Death } & \multirow{2}{*}{ CHR 95\% CI } & \multirow{2}{*}{ AHR $95 \%$ CI } & \multirow{2}{*}{$P$-value } \\
\hline & Yes (\%) & No (\%) & & & \\
\hline Sepsis & $6(1.8)$ & $20(5.9)$ & $3.29(1.03,8.40)^{*}$ & $2.9(1.03,8.40)^{*}$ & 0.045 \\
\hline Hypothermia & $4(1.2)$ & $3(0.9)$ & $12.62(4.27,37.26)^{*}$ & $11.8(3.77,37.02)^{*}$ & $<0.0001$ \\
\hline Hyperthermia & $4(1.2)$ & $13(3.8)$ & $0.321(0.11,0.92)$ & $2.9(0.93,8.98)$ & 0.068 \\
\hline Dehydration & $3(0.9)$ & $212(62.4)$ & $0.757(.337,1.702)$ & $0.5(0.18,1.44)$ & 0.202 \\
\hline Vomiting & $18(5.3)$ & $141(41.5)$ & $1.721(0.83,1.17)$ & $1.6(0.74,3.50)$ & 0.233 \\
\hline Shock & $2(0.6)$ & $5(1.5)$ & $0.277(.07,20.05)$ & $3.8(0.72,20.05)$ & 0.115 \\
\hline Impaired level of consciousness & $3(0.9)$ & $15(4.4)$ & $0.988(0.60,6.56)$ & $2.9(0.83,9.843)$ & 0.096 \\
\hline Not having antibiotics & $23(6.8)$ & $141(41.5)$ & $3.86(1.657,9.015)^{*}$ & $3.7(1.55,8.64)^{*}$ & 0.003 \\
\hline
\end{tabular}

acceptable level of national SAM management guideline at stabilization centers $(<10 \%)[19]$.

The risk of death in children being hypothermic at admission were 11.8 (95\% CI [3.77-37.02]; $p<0.0001)$ times higher than for children with normal temperature. This finding is congruent with the study of Jimma University Specialized Hospital and Dhaka Hospital 3.9 (95\% CI [1.8-8.4.]; 0.001) and 4.8 (95\%CI [2.210.6] 0.01 ) respectively $[3,11]$. This may be due to children with complicated SAM were not isolated in a separate room equipped with radiator to keep the room warm to prevent hypothermia.
Children having sepsis were 2.936 (95\% CI [1.03-8.40], 0.045 ) times more at risk of death when compared with those who have no sepsis. The finding agrees' with research conducted in University of Nigeria Teaching Hospital having sepsis with other co-morbidities shown a significant association with death in SAM patients in SC [4]. In addition, the result reported from Dhaka showed that septic children with complicated SAM were 11.7 ([5.8-23.9] 0.01) times more at risk of death compared with nonseptic [3]. In children with complicated SAM and having sepsis, the risk of dying may be more than double in countries like Ethiopia with limited resources. Because having SAM alone 


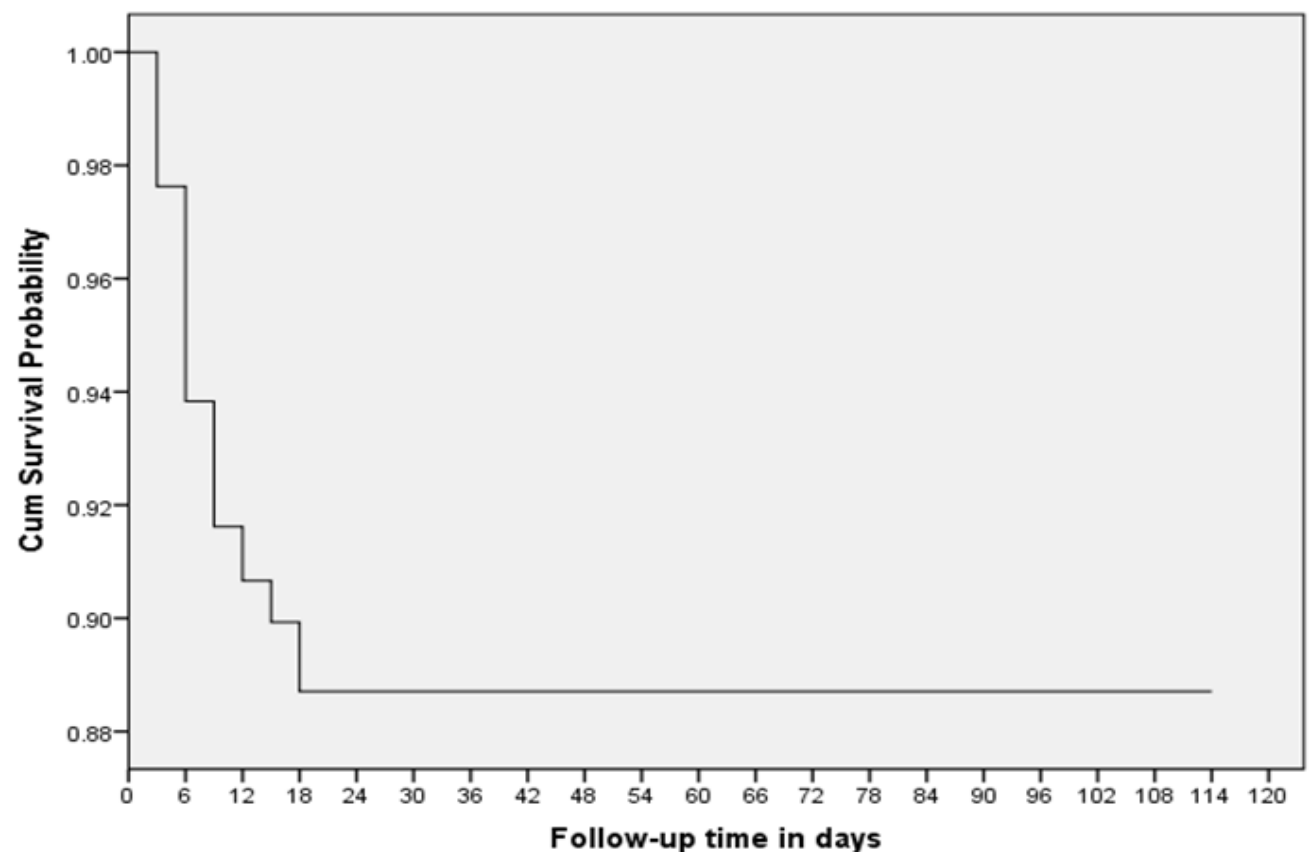

Figure 5: Survival exprience of children with SAM at SC of hospitals in Wolaita Zone January1/ 2013 to March 30/ 2016(n=340)

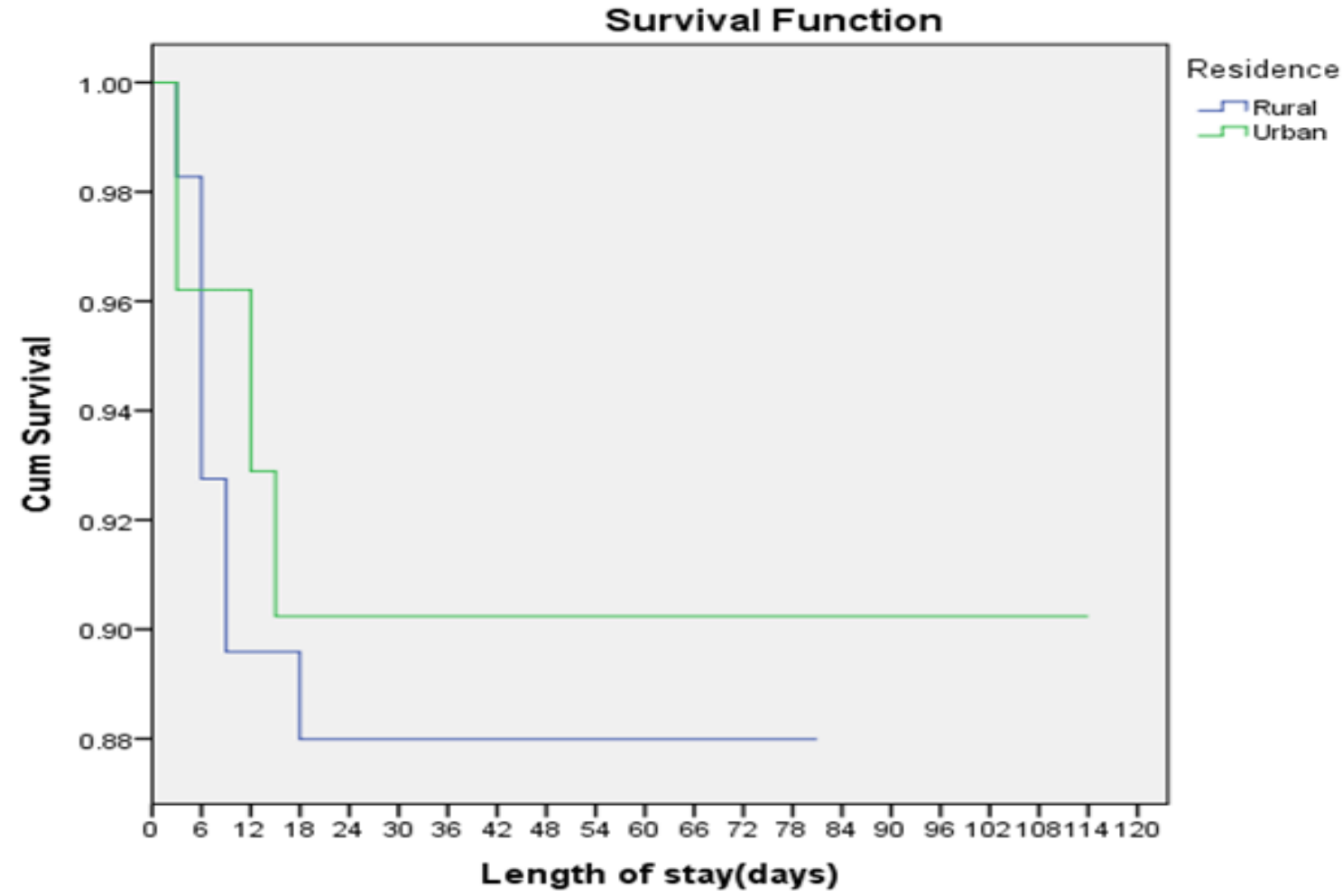

Figure 6: K-M estimates of Survival expriences of complicated SAM children at SC of hospitals by residence,Wolaita Zone January1/ 2013 to March $30 / 2016(n=340)$ 
is enough to compromise the body's immune system, when comorbidities like sepsis added the probability of passing away dramatically increased. Thus, children with sepsis may face more risk of death if not addressed early to mitigate the condition.

On the other hand, children not having antibiotics at admission were by $3.7(95 \% \mathrm{CI}[1.55,8.64] \mathrm{p}=0.003)$ times more likely to die than those had had it. Research conducted in Nigeria contradicting this reported that resistance of enterobacteria to oral antibiotics and sensitivity to respond for third generation cephalosporin drugs [29]. Therefore, it may need another longitudinal study to confirm the drug sensitivity and response.

World Health Organization (WHO) recommends ten basic steps to manage SAM. Managing Hypothermia is the second most important recommendation in children with complicated SAM [30]. Therefore, the factors statistically significant association with the incidence rate of death as mentioned early may be due to poor adherence to guideline for management of SAM and medical complications, performing its management by untrained clinicians and SC setup factors. But this is out of the scope our study and may need a longitudinal prospective study.

\section{Strength and Limitations of Study}

\section{Strengths}

Using cohort study design, reviewing all charts of children admitted with complicated SAM during the study period; who fulfill criteria to confess for inpatient care.

\section{Limitations}

The study was merely based on secondary data, so analysis of risk factors associated with mortality was limited by the information that could be obtained from the patients' charts. Information on various other prognostic factors was either missing or poorly recorded and was of no use for the study.

The incomplete nature of secondary data and inappropriate keeping of old registration books, missing to record some charts by ward log bocks a non estimated limitation and patient's chart made a sample size smaller. Despite these limitations, the study contributes important scientific evidence on factors compromising the survival of children admitted for treatment of complicated SAM in Wolaita.

\section{Conclusion}

The present study identified having sepsis, hypothermia, and not providing antibiotics in children with complicated SAM were identified to be independent predictors of mortality. Preventing hypothermia by isolation of children in a room with warmer and other clothings is indespensible as the guideline advocates. Treating sepsis by providing antibiotics at admission has a major effect in saving early loss of lives of children with complicated SAM in the stabilization centers. Utilization of national SAM management guideline is very poor in the study settings during the study period. However, treatment outcomes (cure rate, death rate, self-discharges, and non-responders) of children aged 0-59 months with complicated SAM that were managed in the hospitals under the study was congruent with national management protocol for SAM.

\section{Recommendations}

Based on the above conclusion, recommendations forwarded to all concerned bodies to decrease/halt early loss of lives of many under-five children with complicated SAM in the SC

- Health care providers working at the SC should give more attention to treat hypothermia by the bases of SAM management protocol.

- Provision of antibiotics at admission for septic children is obligatory in order to decline or halt early death.

- Zonal health department should strengthen the regular training to update the knowledge of SC staffs, death audit system and supply management for further improvement of complicated SAM outcome.

- Federal level guideline should be updated and Policymakers should emphasis on close monitoring and evaluation of the utilization of the guideline and effectiveness of the implementation. In addition, encouraging non-governmental organizations to work on the objectives to reduce the mortality rate due to complicated SAM may also be paramount useful.

\section{Acknowledgments}

First of all I would like to thank almighty God for his support in my entire journey. Then I would like to thank Wolaita Soddo university school of public health for giving me this opportunity to learn and access internet service to search literature for development of this thesis.

Next, my deepest thank goes to my adored advisor Dr. Elasar Tadesse (BSc, MSc, PhD) and Tezera Moshago(MPH) for their unreserved comment, suggestion, support and guidance throughout the development of the thesis.

Also I am highly grateful to Wolaita Zone Health Department, Wolaita Sodo University Teaching and Referral Hospital, Dubbo St. Marry Primary Hospital Administrators for permitting to conduct research in their organization's Stabilization center on Severe Acute Malnutrition.

Finally, I would like to thank my colleagues for their being in touch with me during progression of the whole development of thesis.

\section{Authors' contribution}

Amare Admasu Menta: involved in conception, study design, analysis, interpretation of the results and writing of the manuscript.

Dr. Elazar Tadesse (PhD), Assistant professor of public Health: involved in conception, study design, analysis, interpretation of the results.

Tezera Moshago: Assistant professor of public Health: involved in conception, study design, analysis, interpretation of the results. 
Niguse Mekonnen: Lecturer, data analysis, interpreted and wrote the manuscript. All the authors read and approved the final manuscript.

\section{References}

1. World Health Organization (WHO). Technical aspects of the management of severe acute malnutrition in infants and children. GENEVA:WHO2013.

2. UNICEF. Millennium Development Goals, Goal 4: Reduce Child Mortality 2012

3. Roy SK, Buis M, Weersma R, Khatun W, Chowdhury S, Begum A et al. Risk Factors of Mortality in Severely-malnourished Children Hospitalized with Diarrhoea. J Health Popul Nutr. 2011;29(3):229235.

4. Agozie C Ubesie, Ngozi S Ibeziako, Chika I Ndiokwelu, Chinyeaka M Uzoka, Chinelo A Nwafor. Under-five protein energy malnutrition admitted at the University of in Nigeria Teaching Hospital, Enugu: a 10 year retrospective review. Nutrition Journal. 2012;11:43.

5. Tendai Munthali, Choolwe Jacobs, Lungowe Sitali, Rosalia Dambe, Charles Michelo. Mortality and morbidity patterns in under-five children with severe acute malnutrition (SAM) in Zambia: a five-year retrospective review of hospital-based records (2009-2013). Arch Public Health. 2015;73(1):23. doi: 10.1186/s13690-015-0072-1

6. Oscar Benyers. Outcomes in malnourished children at a tertiary hospital in Swaziland: post implementation of the WHO treatment guideline. University of Pretoria. 2013.

7. Chamois S. Decentralization and scale up of outpatient management of SAM in Ethiopia (2008-2010). Field article. 2011:39-40

8. Francis Lwanga, Rhoda K. Wanyenze, Joseph KB Matovu, Christopher Garimoi Orach. Food Security and Nutritional Status of Children Residing in Sugarcane Growing Communities of East-Central Uganda: A Cross-sectional Study. Journal of Food Security. 2015;3(2):34-39. doi: 10.12691/jfs-3-2-1

9. Tesfalegn T, Tesfaye H, Loha E. Treatment Outcome of Community Based Management of Severe Acute Malnutrition in South Ethiopia: 21st annual public health conference;EPHA;2010.

10. Eklund M, GirmaT. Effectiveness of Integrated Outpatient Care of Severe Acute Malnutrition in Ethiopia. Field Exchange. 2008.

11. Habtemu Jarso, Abdulhalik Workicho, Fessahaye Alemseged. Survival status and predictors of mortality in severely malnourished children admitted to Jimma University Specialized Hospital from 2010 to 2012 Jimma, Ethiopia: a retrospective longitudinal study. BMC Pediatrics. 2015;15:76.

12.Zinabu Tazeze. Treatment outcome and associated factors among under five children with severe acute malnutrition treated at outpatient therapeutic feeding unit in Deghabour hospital, Eastern Ethiopia. 2015;1-56.

13. Abel H. Irena, Mwate Mwambazi, Veronica Mulenga. Diarrhea is Major killer of Children with Severe Acute Malnutrition Admitted to Inpatient Set-up in Lusaka, Zambia. Nutrition Journal. 2011;10:110. doi: 10.1186/1475-2891-10-110

14. Tefera Chane, Lemessa Oljira, Gudina Egata Atomesa, Eskezyiaw Agedew. Treatment Outcome and Associated Factors among UnderFive Children with Severe Acute Malnutrition Admitted to Therapeutic Feeding Unit in Woldia Hospital, North Ethiopia. J Nutr Food Sc 2014;4(6):1-6. doi: 10.4172/2155-9600.1000329
15. Wolaita Zone Health Department(WZHD). Total population of Wolaita Zone. 2015.

16.Wolaita Sodo University. Health Management Information System Annual Report Summary. Sodo2015.

17. Dubbo St. Marry Primary Hospital. Health Management Information System Annual Report Summary. Sodo2015.

18. Federal democratic Republic of Ethiopia MoH. Training course on the management of severe acute malnutrition Second Edition, Addis Ababa, Ethiopia. 2013.

19. Michael G, Yvonne G. Protocol for the Management of Severe Acute Malnutrition. Addis Ababa, Ethiopia: Ethiopian Federal Ministry of Health. 2007.

20. Medical Dictionary. 3rd ed. New Jersey : Wiley Publishing, Inc. Hoboken; 2008.

21. Younas Muhammad, Aqeel Khan Muhammad, Khan Jehanzeb, Shah Faridullah, Munir Arshia. Systematic Analysis Of In-Patients' Care of Severely Malnourished Children at Hayatabad Medical Complex Peshawar: A Tool To Improve Quality Of Care. Gomal Journal of Medical Sciences. 2012;10(1):1-5

22. Abel H Irena, Mwate Mwambazi, Veronica Mulenga. Diarrhea is a Major killer of Children with Severe Acute Malnutrition Admitted to Inpatient Set-up in Lusaka, Zambia. Nutr J. 2011;10:110. doi: 10.1186/1475-2891-10-110

23. United Nations Children's Fund (UNICEF). Evaluation of Community Management of Acute Malnutrition (CMAM): Ethiopia Country Case Study. 2012

24. Maimuna M. Ahmed D (MD). Prevalence of under nutrition, risk factors and outcomes of severe malnutrition among undernourished children admitted to bugando medical centre in mwanza, tanzania [MSc] 2013.

25. Léon G. Blaise Savadogo, Philippe Donnen, Fla Kouéta, Eléonore Kafando, Philippe Hennart, Michèle Dramaix. Impact of HIV/AIDS on mortality and nutritional recovery among hospitalized severely malnourished children before starting antiretroviral teatment Open Journal of Pediatrics. 2013;3(4):340-345. doi:10.4236/ ojped.2013.34061

26. Ashagrie Terefe Abeje, Temesgen Worku Gudayu, Yewunetu Dessalegn Malefia, Birhanu Boru Befftu. Analysis of Hospital Records on Treatment Outcome of Severe Acute Malnutrition: The Case of Gondar University Tertiary Hospital. Pediatrics \& Therapeutics. 2016;6(2):15. doi: 10.4172/2161-0665.1000283

27. Desta Ks. Survival Status and Predictors of Mortality among Children Aged 0-59 Months with Severe Acute Malnutrition Admitted to Stabilization Center at Sekota Hospital Waghemra Zone. J Nutr Disorders Ther. 2015;5(2):1-11. doi: 104172/2161- 05091000160

28. Solomon Amsalu, Zemene Tigabu. Risk factors for severe acute malnutrition in children under the age of five: A case-control study. Ethiop J Health Dev. 2008;22(1):21-25.

29. Page AL, de Rekeneire N, Sayadi S, Aberrane S, Janssens AC, Rieux C et al. Infections in Children Admitted with Complicated Severe Acute Malnutrition in Niger. PLoS One. 2013;8(7): e68699. doi: 10.1371/ journal.pone.0068699

30. Kerak et al. Prevention \& Management of severe acute malnutrition Burden and evidence-based interventions. 2009. 